\title{
Pharmacologic Properties of the Carrier Solutions for Hyperthermic Intraperitoneal Chemotherapy: Comparative Analyses Between Water and Lipid Carrier Solutions in the Rat Model
}

\author{
Eun Jung Park, MD ${ }^{1}$, Junhyun Ahn, PhD $^{2,3}$, Sang Won Gwak ${ }^{2,3}$, Kyung Su Park, $\mathrm{PhD}^{4}$, Seung Hyuk Baik, MD, \\ $\mathrm{PhD}^{1}$, and Sung-Joo Hwang, $\mathrm{PhD}^{2,3}$
}

${ }^{1}$ Division of Colon and Rectal Surgery, Department of Surgery, Gangnam Severance Hospital, Yonsei University College of Medicine, Seoul, Korea; ${ }^{2}$ Yonsei Institute of Pharmaceutical Sciences, Yonsei University, Incheon, Korea; ${ }^{3}$ College of Pharmacy, Yonsei University, Incheon, Korea; ${ }^{4}$ Advanced Analysis Center, Korea Institute of Science and Technology, Seoul, Korea

\begin{abstract}
Background. Carrier solutions play an important role in the distribution, plasma absorption, chemical stability, and solubility of anticancer agents during hyperthermic intraperitoneal chemotherapy (HIPEC). In the current study, lipophilic properties of carrier solutions were evaluated to determine whether they improved anticancer drug absorption rates using mitomycin-C (MMC) or oxaliplatin HIPEC as compared to hydrophilic carrier solutions.

Methods. Sprague-Dawley rats were divided into two groups: MMC and oxaliplatin treatment groups. Each group was then further subdivided by carrier solution: Dianeal ${ }^{\circledR}$ PD-2 peritoneal dialysis solution, 5\% dextrose solution and $20 \%$ lipid solution (Lipision ${ }^{\circledR}$ ). HIPEC was
\end{abstract}

The results about mitomycin- $\mathrm{C}$ in this article were presented at the poster presentation at the 10th International Congress on Peritoneal Surface Malignancy (PSOGI) 2016 Biannual Meeting, Washington DC, USA.

Electronic supplementary material The online version of this article (https://doi.org/10.1245/s10434-018-6628-x) contains supplementary material, which is available to authorized users.

(C) The Author(s) 2018

First Received: 30 October 2017;

Published Online: 18 July 2018

S. H. Baik, MD, $\mathrm{PhD}$

e-mail: whitenoja@yuhs.ac

S.-J. Hwang, PhD

e-mail: sjh11@yonsei.ac.kr performed over $60 \mathrm{~min}$ at $41-42{ }^{\circ} \mathrm{C}$ using the anticancer drugs MMC $\left(35 \mathrm{mg} / \mathrm{m}^{2}\right)$ or oxaliplatin $\left(460 \mathrm{mg} / \mathrm{m}^{2}\right)$. The plasma area under the curve (AUC; $\mathrm{AUC}_{\text {plasma }}$ ), peritoneal AUC $\left(\mathrm{AUC}_{\text {peritoneum }}\right)$, and peritoneal/plasma AUC ratios were compared among HIPEC carrier solutions.

Results. Plasma drug concentrations were significantly different among carrier solutions, varying by time. In contrast, peritoneal drug concentrations did not change with carrier solution. In the MMC group, the peritoneal/plasma AUC ratio of a lipid solution was three times higher than Dianeal ${ }^{\circledR}(p<0.001)$. In the oxaliplatin group, the peritoneal/plasma AUC ratio was significantly different between carrier solutions $(p=0.046)$. Although the oxaliplatin $\mathrm{AUC}_{\text {peritoneum }} \mathrm{did}$ not vary $(p=0.941)$, the $\mathrm{AUC}_{\text {plasma }}$ of a lipid solution was lower than that of $5 \%$ dextrose solution $(p=0.039)$.

Conclusions. The lipid carrier solution increases the peritoneal/plasma AUC ratio and decreases plasma absorption rates. However, further study is required before clinical uses, considering its pharmacologic properties and possible risks after HIPEC.

Hyperthermic intraperitoneal chemotherapy (HIPEC) after cytoreductive surgery is utilized based on studies showing that microscopic residual tumors can be eradicated by intraperitoneally (IP)-administrated anticancer drugs, with enhanced cytotoxic effect at $41-43{ }^{\circ} \mathrm{C} .{ }^{1,2}$ As IP drug administration results in a high concentration gradient in the peritoneal-plasma barriers, anticancer drugs can infiltrate tumors following the principles of convection, 
diffusion, and recirculation, which is different from systemic chemotherapy administration. ${ }^{1,3,4}$ High drug concentration within the peritoneal cavity produces a driving force for anticancer drugs to infiltrate tumor cells during HIPEC. ${ }^{3}$ In addition, slow plasma absorption rates of anticancer drugs enhance IP drug efficacy and reduce systemic toxicities. ${ }^{5}$

Anticancer drug tissue penetration during HIPEC depends on several pharmacologic properties, including drug concentration, time of exposure, molecular weight (MW), temperature, and lipophilicity. ${ }^{6}$ For optimal HIPEC treatment, it is crucial to select the appropriate carrier solution for each anticancer drug to enhance drug activity, because pharmacologic properties are uniquely different during use in the peritoneal cavity. ${ }^{6,7}$ Especially, carrier solutions have an important role in the distribution, plasma absorption, chemical stability, and solubility of anticancer agents during HIPEC. ${ }^{8,9}$ Previous reports evaluated hydrophilic carrier solution pharmacologic properties for HIPEC. ${ }^{6,10-12}$ In the current study, the ability of lipophilic carrier solutions to improve the absorption rate of anticancer drugs during HIPEC was determined. In addition, the pharmacologic characteristics of mitomycin C (MMC) and oxaliplatin were evaluated according to HIPEC carrier solution to find the optimal pharmacologic conditions to treat patients with colorectal cancer carcinomatosis.

\section{METHODS}

\section{Experimental Design}

According to anticancer treatment, animals were divided into two groups: MMC or oxaliplatin HIPEC. Three carrier solutions were evaluated in this study: Dianeal ${ }^{\circledR}$ PD-2 $1.5 \%$ peritoneal dialysis solution (Baxter, USA), 5\% dextrose solution, and $20 \%$ lipid solution $\left(\right.$ Lipision ${ }^{\circledR}$, JW Pharmaceutical, Republic of Korea). Three rats were used to perform HIPEC for each carrier solution. The Animal Research Committee of Ajou University, Republic of Korea (IACUC Number 2016-0029) approved the study protocol. Experiments were performed at the Laboratory Animal Research Center at Ajou University Medical Center, Suwon, Korea.

\section{Animals}

Fifteen, 8-week-old, male Sprague-Dawley rats, weighing 290-320 g, were purchased from Orientbio Inc. (Kyunggi-do, Korea). Mean body surface area (BSA) was calculated by using the Du Bois method. ${ }^{13}$ Rats were housed in filter-top cages for 1 week before experiments with free access to food and water (Ziegler ${ }^{\circledR}$ lab animal diet, USA). The animal laboratory was kept under standard conditions with temperature $21-24{ }^{\circ} \mathrm{C}$, humidity $40-60 \%$, 12-h light cycle, and filtered air. A total of 15 rats, comprising 3 rats per group, was used in this study.

\section{Experimental Settings for HIPEC}

The HIPEC experimental equipment was setup as shown in Fig. 1. The inflow line was inserted into the roller of a peristaltic pump (Masterflex C/L pump ${ }^{\circledR}$, Bernant, USA) and delivered HIPEC solutions at a flow rate of $40 \mathrm{~mL} /$ $\min$. The outflow line connected the abdominal cavity to a reservoir chamber. Outflow line circulating fluids could be returned to the reservoir chamber using negative pressure induced by suction. The temperature of circulating HIPEC solutions was maintained at $41-42{ }^{\circ} \mathrm{C}$. Both inflow and outflow lines were heated by a circulating warm bath (Lauda E100 ${ }^{\circledR}$, Lauda, Germany). Three sites were monitored for consistent temperature using thermometers: circulating HIPEC solutions in the rat abdominal cavity and rectum, and the heated water in the warm bath.

\section{Hyperthermic Intraperitoneal Chemotherapy (HIPEC) Procedure}

All animals received general inhalation anesthesia using $3 \%$ isoflurane with 1:1 oxygen and nitrous oxide before HIPEC procedures. Before the anesthesia, $20 \mathrm{~mL}$ of water was given orally to all rats to prevent dehydration. According to the Coliseum technique, a 4- to 5-cm medial longitudinal incision was made in the rat abdominal wall. ${ }^{14}$ Then, all margins of the abdominal wall were elevated and fixed in the acryl plates, which were located $15-\mathrm{cm}$ above the basal plate. After setting the HIPEC equipment as described in Fig. 1, HIPEC solutions were prepared with MMC $\left(35 \mathrm{mg} / \mathrm{m}^{2}\right)$ or oxaliplatin of $\left(460 \mathrm{mg} / \mathrm{m}^{2}\right)$, which were mixed with $300 \mathrm{~mL}$ of carrier solution. Blood and peritoneal fluid samples were collected at 0 (starting time), 5, 10, 20, 30, 45, and $60 \mathrm{~min}$ after beginning HIPEC. Peritoneal fluid was collected in the HIPEC circulated fluid of the abdominal cavity, and blood samples were collected from the retro-orbital venous sinus after inhalation anesthesia. All samples were kept frozen at $-60{ }^{\circ} \mathrm{C}$ until further analyses.

\section{Sample Preparation and Analytical Methods}

Samples were thawed at room temperature before analyzing drug concentration. Protein precipitation was performed to remove blood and peritoneal components. For MMC analysis, samples were centrifuged at 15,000 rpm at $4{ }^{\circ} \mathrm{C}$ for $15 \mathrm{~min}$. Then, $75 \mu \mathrm{L}$ of the supernatant was diluted with $90 \%$ acetonitrile $(300 \mu \mathrm{L})$. After vortex 


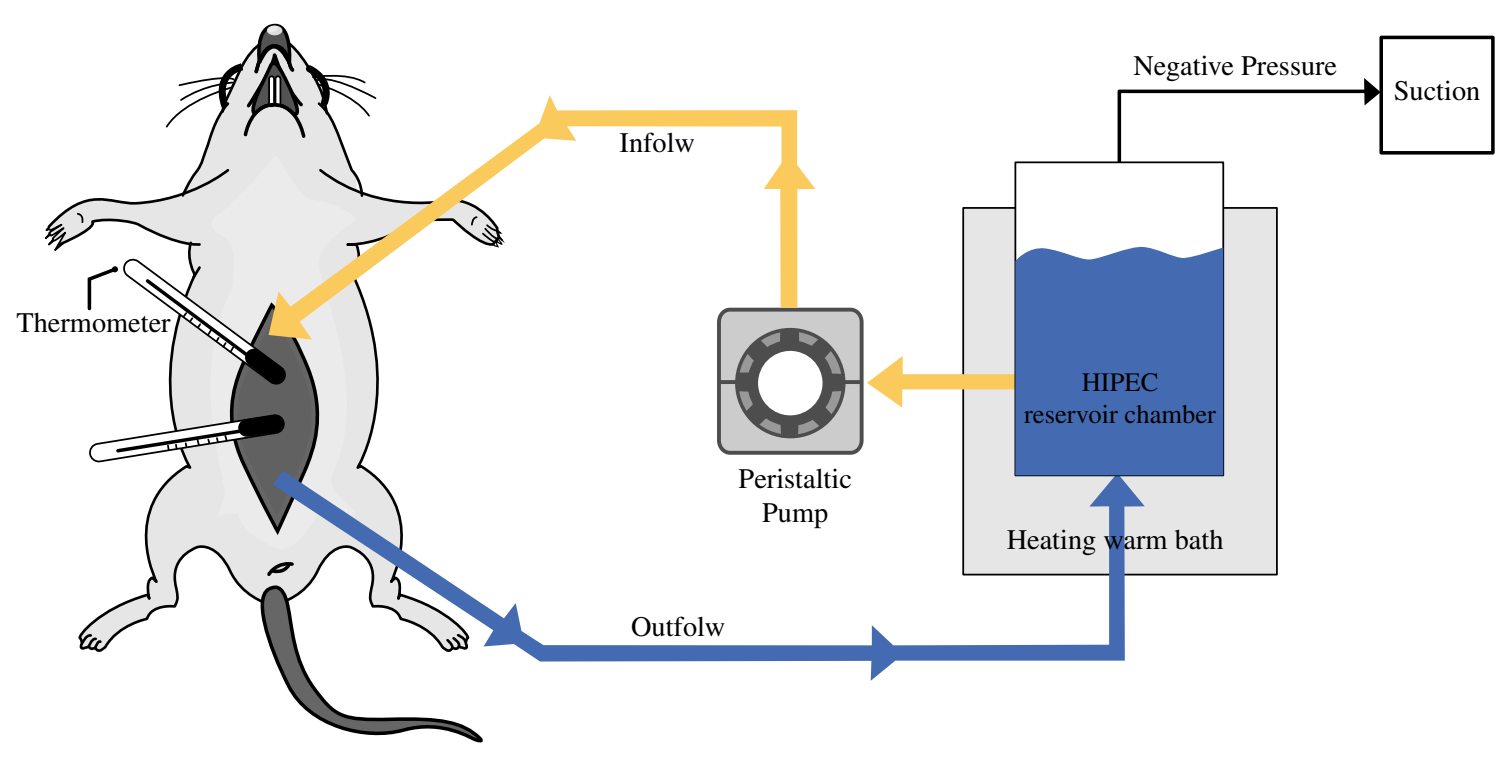

FIG. 1 HIPEC rat model

shaking for $10 \mathrm{~min}$ at room temperature, samples were centrifuged again at $15,000 \mathrm{rpm}, 4{ }^{\circ} \mathrm{C}$ for $15 \mathrm{~min}$. After removing the organic solvent layer and precipitated proteins, supernatants were leached with a $0.2-\mu \mathrm{m}$ syringe filter. The filtered supernatants were then analyzed by tandem mass spectrometry (Agilent 6490 QQQ Triple Quadrupole LC/MS System ${ }^{\circledR}$, Agilent Technologies, Santa Clara, CA) to measure MMC drug concentrations.

Oxaliplatin solutions were analyzed by inductively coupled plasma-quadrupole mass spectrometry (ICP-QMS; NexION 300D ${ }^{\circledR}$, PerkinElmer, US). To measure platinum complex in the oxaliplatin, samples were prepared using a microwave digestion system. Samples were placed in Teflon vessels with $5 \mathrm{~mL}$ of $\mathrm{HNO}_{3}$ and digested in a microwave oven at $800 \mathrm{~W}$ for $1 \mathrm{~h}$. After diluting to $25 \mathrm{~mL}$ with distilled water, samples were analyzed by ICP-QMS.

\section{Statistical Analyses}

Statistical analyses were performed using SPSS 23 (SPSS Inc., Chicago, IL), SAS 9.3 (SAS Institute Inc., Cary, NC), and R 3.4.1 software (The R Foundation for Statistical Computing). Linear mixed models were used to compare drug concentrations between carrier solutions according to time. The independent $t$ test and one-way analysis of variance (ANOVA) were used to compare area under the curve (AUC) ratios among carrier solutions. Post-hoc analyses were performed using the Scheffe correction method. A $p$ value $<0.05$ was considered statistically significant.

\section{RESULTS}

Comparison of Drug Concentrations over Time During HIPEC

In the MMC group, the plasma drug concentration in the Dianeal ${ }^{\circledR}$ group was increased compared to the lipid solution over time, as shown in Table 1 and Fig. 2a. However, MMC concentrations in the peritoneal fluid were not significantly different between Dianeal ${ }^{\circledR}$ and lipid solutions $(p=0.2313)$.

In the oxaliplatin group, the plasma drug concentrations were significantly different among the carrier solutions by time $(p=0.0049)$. The plasma absorption rate was highest in the oxaliplatin mixed with $5 \%$ dextrose solution group (Fig. 2c). However, oxaliplatin concentrations in the peritoneal fluid were not significantly different among the carrier solutions (Table 1).

\section{Comparison of AUC Ratios Between Water and Lipid Solutions}

In the MMC group, the AUC of the peritoneal fluid $\left(\mathrm{AUC}_{\text {peritoneum }}\right)$ was higher in the lipid carrier solution than Dianeal ${ }^{\circledR}$. Conversely, the AUC of the plasma $\left(\mathrm{AUC}_{\text {plasma }}\right)$ was lower in the lipid solution compared to Dianeal ${ }^{\circledR}$. Thus, the peritoneal/plasma AUC ratio of the lipid carrier solution was approximately three times higher than Dianeal ${ }^{\circledR}$ in the MMC group $(p<0.001$; Table 2$)$.

In the oxaliplatin group, the $\mathrm{AUC}_{\text {peritoneum }}$ was not significantly different among the carrier solutions $(p=0.941)$. However, the $\mathrm{AUC}_{\text {plasma }}$ of the $5 \%$ dextrose solution was 


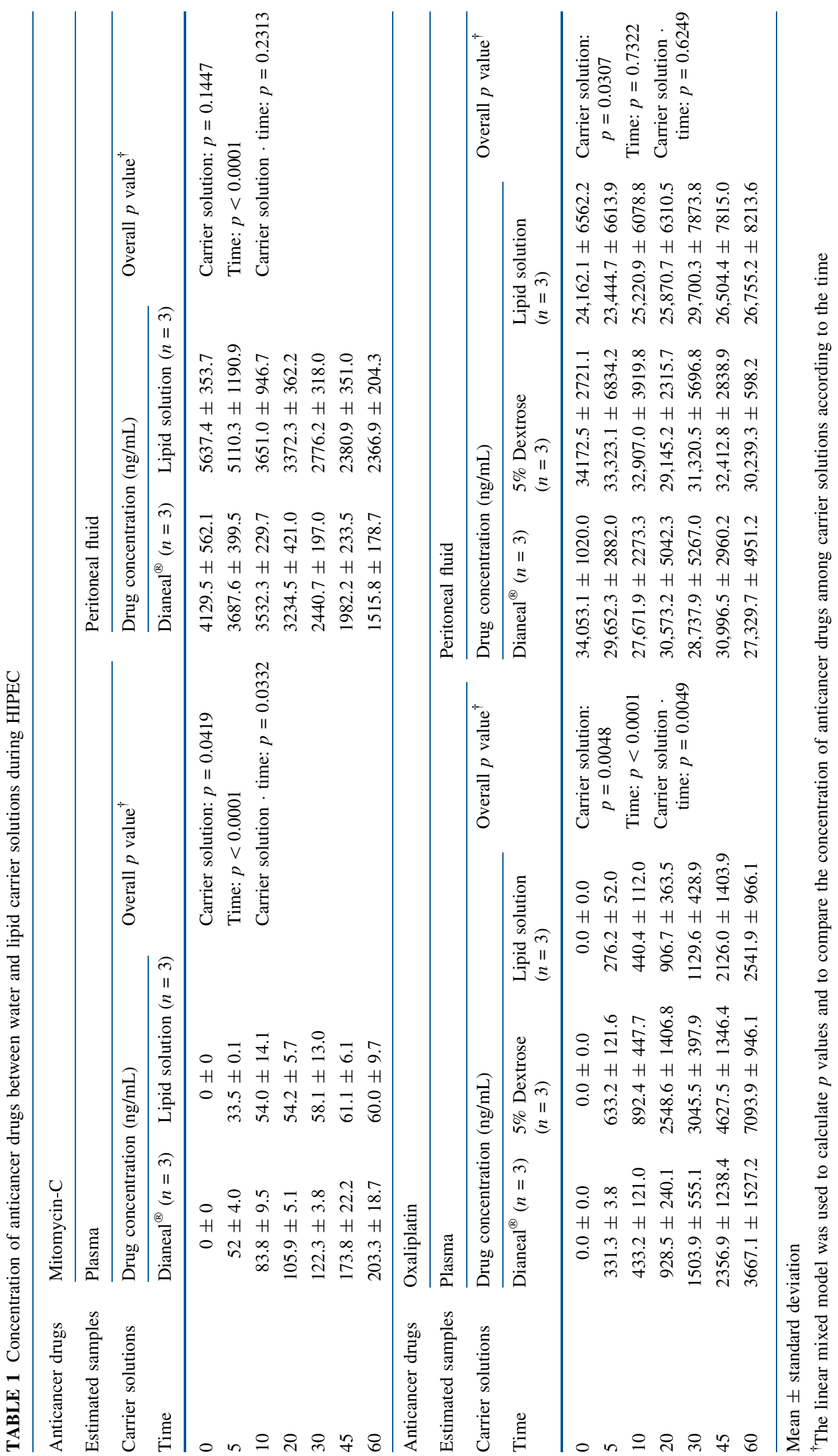


(a)

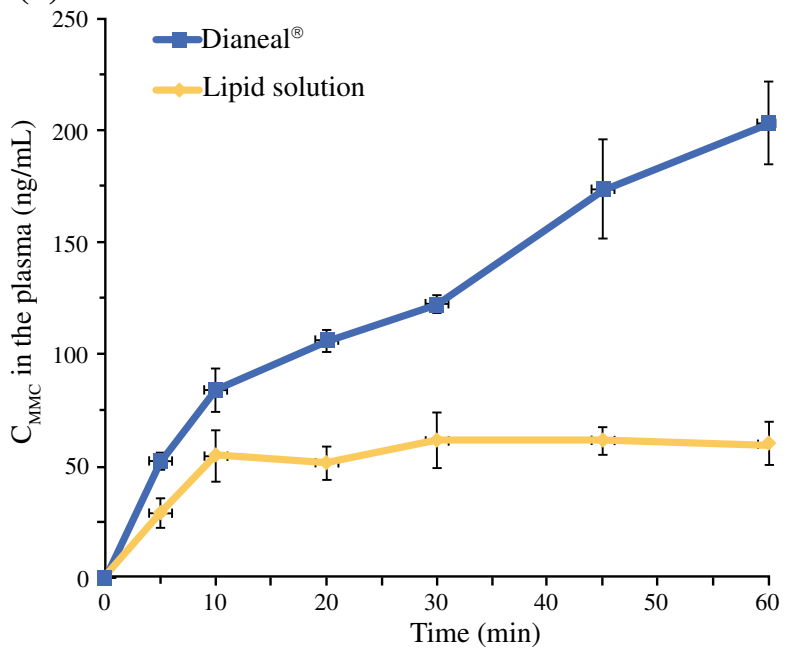

(c)

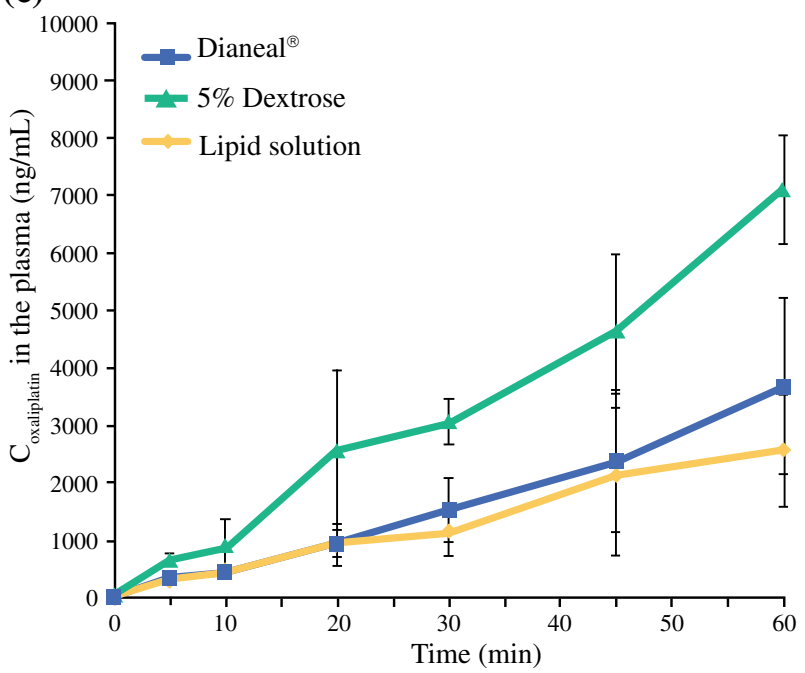

FIG. 2 Concentrations of mitomycin-C (MMC) and oxaliplatin by HIPEC carrier solution. The concentration of MMC in plasma (a) and in the peritoneal fluid (b). The concentration of oxaliplatin in the

higher than that of the lipid solution $(p=0.039)$. There was no significant difference in the $\mathrm{AUC}_{\text {plasma }}$ between the $5 \%$ dextrose solution and Dianeal ${ }^{\circledR}(p=0.070)$. The oxaliplatin AUC ratio was different among the carrier solutions. In particular, the oxaliplatin AUC ratio in the lipid solution was marginally higher than that of the $5 \%$ dextrose solution during HIPEC $(p=0.056)$. According to AUC ratios of oxaliplatin, which were cut off at $30 \mathrm{~min}$, AUC ratio of lipid solution was higher than $5 \%$ dextrose solution (Supplementary Table 1).

\section{Plasma Drug Concentration Gradient by Carrier Solution}

To estimate the changes in plasma drug concentration among the carrier solutions, the plasma concentration (b)

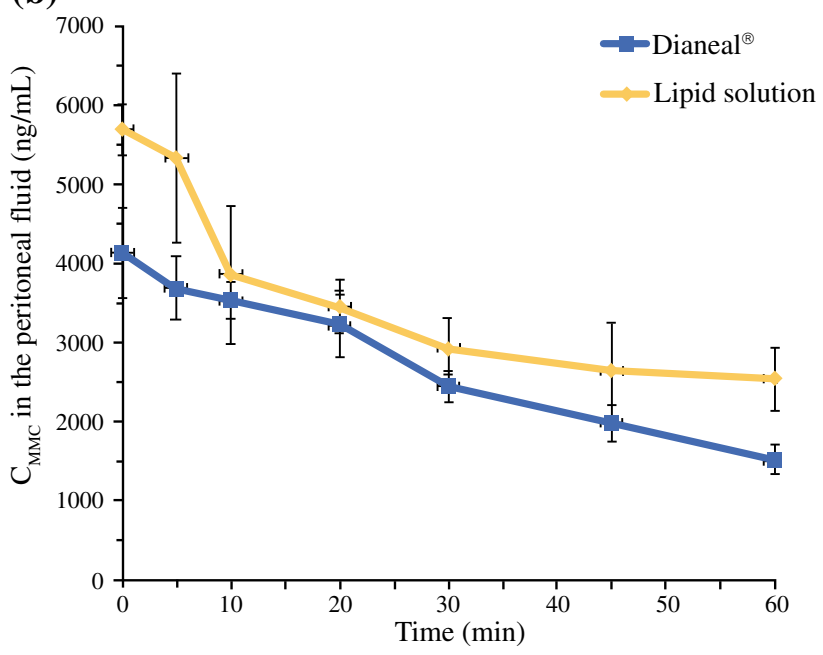

(d)

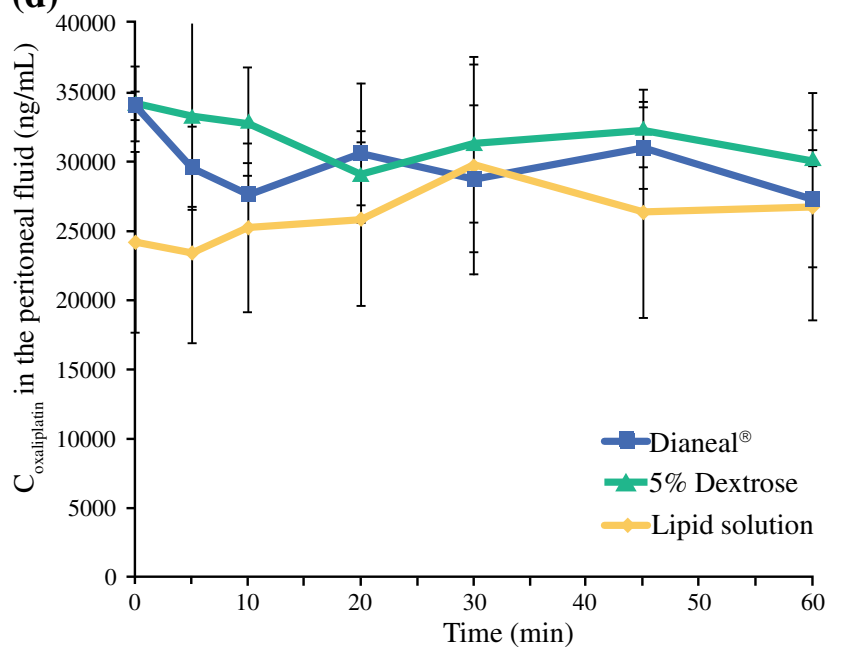

plasma (c) and in the peritoneal fluid (d). $C_{\mathrm{MMC}}$, concentration of MMC; $C_{\text {oxaliplatin, }}$ concentration of oxaliplatin

gradient of anticancer drugs was calculated using a linear mixed model, according to time. As shown in Table 3, the estimated plasma concentration gradient of MMC had a steeper slope with Dianeal ${ }^{\circledR}$ compared with the lipid solution. On the other hand, the estimated plasma concentration gradient of oxaliplatin had a steeper slope in the $5 \%$ dextrose solution compared to both the Dianeal ${ }^{\circledR}$ and the lipid solution $(p<0.001)$. However, there was no significant difference in the oxaliplatin estimated plasma concentration gradient between the Dianeal ${ }^{\circledR}$ and lipid solutions.

\section{DISCUSSION}

HIPEC has pharmacologic principles that provide regionally intensified antineoplastic drug concentration in the peritoneal cavity and promote tumor cell penetration 
with a prolonged presence in the peritoneal-plasma barrier. $^{3}$ Based on the anatomical structures of the peritoneum, anticancer drugs that have large MW and water insolubility are correlated with a larger AUC ratio and longer stay in the peritoneal cavity. ${ }^{1}$ The intercellular gaps of the mesothelium are larger than those in the endothelium; therefore, large molecules that cannot pass through endothelial layers do penetrate mesothelial layers. The MW of MMC is $334.3 \mathrm{~g} / \mathrm{mol}$ and that of oxaliplatin is $397.3 \mathrm{~g} / \mathrm{mol}$, both smaller than anticancer drugs paclitaxel $(\mathrm{MW}=853.9 \mathrm{~g} / \mathrm{mol}) \quad$ and docetaxel $\quad(\mathrm{MW}=861.9$ $\mathrm{g} / \mathrm{mol}) .{ }^{4}$ In addition, the logarithm ratio of partition coefficient $(\log P)$ of MMC is -1.6 and oxaliplatin is -0.47 , which tends to be water-soluble. Therefore, these pharmacologic characteristics of both MMC and oxaliplatin are not inherently suitable to enhance the effect of IP chemotherapy, with the exception of water solubility, which is a useful characteristic to circulate solutes during HIPEC.

However, importantly, our results demonstrate that the use of a lipid carrier solution increased the AUC ratio and reduced the plasma absorption rate. Although the oxaliplatin $\mathrm{AUC}_{\text {peritoneum }}$ was not significantly different among carrier solutions, our data showed that a lipid carrier solution has advantages, controlling the permeability of endothelial layers and reducing the plasma absorption rate during HIPEC. This could be a result of hydrophobic lipid particles that are resistant to traversing the plasma membrane of endothelial cell layers.

In HIPEC treatment of colorectal cancer patients with peritoneal carcinomatosis, both MMC and oxaliplatin are used. The MMC carrier solution currently used in most HIPEC centers is an isotonic solution. ${ }^{15}$ Therefore, in this study, we compared the effects of carrier solutions except $5 \%$ dextrose solution in the MMC HIPEC. However, the optimal selection of carrier solution during oxaliplatin HIPEC is still debated, as oxaliplatin can be degraded in chloride-containing carrier solutions, because the oxalate ligand of oxaliplatin can be substituted into chloride ions. ${ }^{12}$ Elias et al. ${ }^{16}$ reported favorable oncological outcomes using oxaliplatin with 5\% dextrose carrier solution. However, it has been reported that the hypotonicity of the dextrose solution increases the risk of postoperative complications after HIPEC, such as severe electrolyte imbalance, hyperglycemia, tissue edema, and intraperitoneal hemorrhagic complications. ${ }^{10,17-19}$ Therefore, in the current study, we compared the oxaliplatin AUC ratios among three carrier solutions: $5 \%$ dextrose solution, Dianeal ${ }^{\circledR}$, and lipid solution to compare lipophilicity versus hydrophilicity and chloride-containing versus non-chloride-containing solutions. 
TABLE 3 Estimated formulas for the anticancer drug concentration in the plasma

\begin{tabular}{|c|c|c|c|c|}
\hline Anticancer drugs & Carrier solutions & $\begin{array}{l}\text { Estimated formulas of the graph } \\
\text { for the plasma drug concentrations } \\
\text { (MMC: Fig. 2a, oxaliplatin: Fig. 2c) }\end{array}$ & $\begin{array}{l}\text { Estimated concentration } \\
\text { gradient (SE) }\end{array}$ & $p$ value $^{\dagger}$ \\
\hline \multirow[t]{2}{*}{ Mitomycin-C } & Dianeal $^{\circledR}$ & $C_{\text {plasma }}=3.05 \cdot$ time +30.99 & $3.05(0.21)$ & \multirow[t]{2}{*}{$<0.0001$} \\
\hline & Lipid solution & $C_{\text {plasma }}=0.74 \cdot$ time +27.80 & $0.74(0.23)$ & \\
\hline \multirow[t]{3}{*}{ Oxaliplatin } & Dianeal $^{\circledR}$ & $C_{\text {plasma }}=59.15 \cdot$ time -126.21 & $59.15(7.52)$ & $<\mathbf{0 . 0 0 0 1}$ \\
\hline & $5 \%$ dextrose solution & $C_{\text {plasma }}=113.21 \cdot$ time -57.74 & $113.21(7.35)$ & \multirow{2}{*}{$\begin{array}{l}\text { (Dianeal vs. 5DW, } p<0.001 \\
\text { Dianeal vs. lipid, } p=0.3957 \text {; } \\
\text { 5DW vs. lipid, } p<0.001 \text { ) }\end{array}$} \\
\hline & Lipid solution & $C_{\text {plasma }}=43.05 \cdot$ time +14.56 & $43.05(7.35)$ & \\
\hline
\end{tabular}

Estimated formulas was calculated by random intercept model

Bold values are statistically significant $(p<0.05)$

$C_{\text {plasma }}$, concentration of plasma; SE, standard error; $5 \mathrm{DW}, 5 \%$ dextrose

${ }^{\dagger}$ Calculated by linear mixed model

According to the comparison of carrier solutions between lipophilicity and hydrophilicity, the oxaliplatin $\mathrm{AUC}_{\text {peritoneum }}$ was not significantly different among carrier solutions. However, the $\mathrm{AUC}_{\text {plasma }}$ of the lipid solution was lower than that of the 5\% dextrose solution. In addition, the AUC ratio in the lipid solution was marginally higher than hydrophilic carrier solution in both oxaliplatin and MMC HIPEC. Thus, in this study, the lipophilicity of a carrier solution seemed to have an advantage in reducing plasma absorption and increasing the AUC ratio compared with hydrophilic carrier solutions.

In HIPEC using oxaliplatin, 30-min duration is regarded as clinically suitable considering half-life of oxaliplatin and systemic toxicities. ${ }^{20}$ However, because this study was the first experiment to use a lipophilic carrier solution for oxaliplatin-HIPEC, HIPEC was performed to evaluate fully pharmacologic properties of lipid carrier solution until $60 \mathrm{~min}$. The AUC ratio of oxaliplatin in the lipid solution was higher than 5\% dextrose solution at both 30 and $60 \mathrm{~min}$.

Compared with the pharmacological effects of oxaliplatin in the chloride-containing solutions, our results support the effectiveness of Dianeal ${ }^{\circledR}$. The chloride concentration of Dianeal ${ }^{\circledR}$ is $96 \mathrm{mmol} / \mathrm{L}$, whereas that for $5 \%$ dextrose solution is $0 \mathrm{mmol} / \mathrm{L}$. Lipision ${ }^{\circledR}$ is composed of purified soybean oil, purified phospholipid, and glycerin. As demonstrated in Table 2, Dianeal ${ }^{\circledR}$ exhibited advantages to reducing plasma absorption of anticancer drugs, compared with $5 \%$ dextrose solution, when performing oxaliplatin HIPEC. In addition, structural instability of oxaliplatin in the chloride-containing solutions during HIPEC could be acceptable, as a previous report by Mehta et al. ${ }^{12,21}$ indicated that the degradation rates of oxaliplatin is limited within $10-15 \%$ in the chloride-containing HIPEC carrier solution. It is also expected that peritoneal dialysis solutions such a Dianeal ${ }^{\circledR}$ can have advantages to reduce postoperative complications, such as electrolyte imbalance and metabolic disturbance.
Our study results also showed that the AUC ratio of a lipid carrier solution was larger than other carrier solutions. However, there are some limitations in using lipid carrier solutions in clinical applications for HIPEC. Lipision ${ }^{\circledR}$, which was used in this study, is a fat emulsion. Although the lipid layers of Lipision ${ }^{\circledR}$ retard plasma absorption rates of anticancer drugs during HIPEC, the hypertonicity and electrical resistance of these lipid layers can inhibit the permeability of peritoneal-plasma barriers. In addition, according to the pharmacokinetic principles of HIPEC, longer duration in the peritoneum delays recirculation of the tumor core. ${ }^{1}$ Because the efficacy of HIPEC is related to sustained peritoneal drug concentrations, as well as drug infiltration into the tumor core, this contrary phenomenon should be considered in selecting an optimal IP chemotherapeutic agent. In our results, although the lipid carrier solution prolonged peritoneal occupancy, as well as reduced plasma absorption rate, the hydrophobicity of the lipid solution might be inadequate to recirculate into tumor core from the capillary vessels and to increase cytotoxicity. Furthermore, because the bioavailability of anticancer agents is assessed from the release rate of an entrapped drug in lipid layers, it can be questioned whether anticancer agents mixed in a lipid carrier solution have complete tumor cell cytotoxicity during HIPEC. ${ }^{3,22,23}$ The release rate of anticancer drugs in the lymphatic channels and the risk of fat embolism are also to be considered when a lipid carrier solution is used during HIPEC.

This study has several limitations, including a small sample-sized experiment in the animal model. In addition, there is a lack of investigation into both the cytotoxic effects and the rate of lymphatic spread of anticancer drugs during HIPEC with a lipophilic carrier solution. Naïve oxaliplatin is known to have less cytotoxicity than dichloro-platinum compound $\mathrm{Pt}(\mathrm{dach}) \mathrm{Cl}_{2}$, which is an active form that is transformed in chloride-containing media. $^{24,25}$ Because our study measured platinum 
concentration of oxaliplatin, further studies are required to measure the concentration of oxaliplatin transformation to understand both structural instability and cytotoxicity of oxaliplatin depending on different HIPEC carrier solutions.

\section{CONCLUSIONS}

A lipid carrier solution is promising, because it increases the AUC ratio and decreases plasma absorption during HIPEC. However, 5\% dextrose solution is inferior to both Dianeal ${ }^{\circledR}$ and lipid solutions as an oxaliplatin-based HIPEC carrier solution. The choice between Dianeal ${ }^{\circledR}$ and lipid solutions should be made based on considerations of safety and further data regarding the actual efficacy of cytotoxic agents in these solutions.

ACKNOWLEDGEMENT This study was supported by a Faculty Research Grant of Yonsei University College of Medicine for 2015 (6-2015-0055) and Basic Science Research Program through the National Research Foundation of Korea (NRF) funded by the Ministry of Science and ICT (NRF-2017R1A2B2011520).

CONFLICT OF INTEREST The authors have no conflicts of interest or financial ties of disclose.

OPEN ACCESS This article is distributed under the terms of the Creative Commons Attribution 4.0 International License (http://crea tivecommons.org/licenses/by/4.0/), which permits unrestricted use, distribution, and reproduction in any medium, provided you give appropriate credit to the original author(s) and the source, provide a link to the Creative Commons license, and indicate if changes were made.

\section{REFERENCES}

1. Fujiwara K, Armstrong D, Morgan M, Markman M. Principles and practice of intraperitoneal chemotherapy for ovarian cancer. Int J Gynecol Cancer. 2007;17(1):1-20.

2. Ceelen WP, Flessner MF. Intraperitoneal therapy for peritoneal tumors: biophysics and clinical evidence. Nat Rev Clin Oncol. 2010;7(2):108-15.

3. De Smet L, Ceelen W, Remon JP, Vervaet C. Optimization of drug delivery systems for intraperitoneal therapy to extend the residence time of the chemotherapeutic agent. Scientific World J. 2013. https://doi.org/10.1155/2013/720858.

4. Yan TD, Cao CQ, Munkholm-Larsen S. A pharmacological review on intraperitoneal chemotherapy for peritoneal malignancy. World J Gastrointest Oncol. 2010;2(2):109-16.

5. Sugarbaker PH, Van der Speeten K. Surgical technology and pharmacology of hyperthermic perioperative chemotherapy. $J$ Gastrointest Oncol. 2016;7(1):29-44.

6. Mohamed F, Marchettini P, Stuart OA, Sugarbaker PH. Pharmacokinetics and tissue distribution of intraperitoneal paclitaxel with different carrier solutions. Cancer Chemother Pharmacol. 2003;52(5):405-10.

7. Mohamed F, Sugarbaker PH. Carrier solutions for intraperitoneal chemotherapy. Surg Oncol Clin N Am. 2003;12(3):813-24.

8. Kusamura S, Dominique E, Baratti D, Younan R, Deraco M. Drugs, carrier solutions and temperature in hyperthermic intraperitoneal chemotherapy. J Surg Oncol. 2008;98(4):247-52.
9. Van der Speeten K, Stuart OA, Sugarbaker PH. Pharmacokinetics and pharmacodynamics of perioperative cancer chemotherapy in peritoneal surface malignancy. Cancer J. 2009;15(3):216-24.

10. Elias D, Raynard B, Bonnay M, Pocard M. Heated intra-operative intraperitoneal oxaliplatin alone and in combination with intraperitoneal irinotecan: pharmacologic studies. Eur J Surg Oncol. 2006;32(6):607-13.

11. Elias D, Matsuhisa T, Sideris L, et al. Heated intra-operative intraperitoneal oxaliplatin plus irinotecan after complete resection of peritoneal carcinomatosis: pharmacokinetics, tissue distribution and tolerance. Ann Oncol. 2004;15(10):1558-65.

12. Mehta AM, Van den Hoven JM, Rosing H, et al. Stability of oxaliplatin in chloride-containing carrier solutions used in hyperthermic intraperitoneal chemotherapy. Int $J$ Pharm. 2015;479(1):23-7.

13. Du Bois D, Du Bois EF. A formula to estimate the approximate surface area if height and weight be known. 1916. Nutrition. 1989;5(5):303-11; discussion 312-3.

14. Sugarbaker PH, Averbach AM, Jacquet P, Stephens AD, Stuart OA. A simplified approach to hyperthermic intraoperative intraperitoneal chemotherapy (HIIC) using a self-retaining retractor. Cancer Treat Res. 1996;82:415-21.

15. Verwaal VJ, van Ruth S, de Bree E, et al. Randomized trial of cytoreduction and hyperthermic intraperitoneal chemotherapy versus systemic chemotherapy and palliative surgery in patients with peritoneal carcinomatosis of colorectal cancer. J Clin Oncol. 2003;21(20):3737-43.

16. Elias D, Lefevre JH, Chevalier J, et al. Complete cytoreductive surgery plus intraperitoneal chemohyperthermia with oxaliplatin for peritoneal carcinomatosis of colorectal origin. J Clin Oncol. 2009;27(5):681-5.

17. Charrier T, Passot G, Peron J, et al. Cytoreductive surgery combined with hyperthermic intraperitoneal chemotherapy with oxaliplatin increases the risk of postoperative hemorrhagic complications: analysis of predictive factors. Ann Surg Oncol. 2016;23(7):2315-22.

18. Ceelen WP, Peeters M, Houtmeyers P, Breusegem C, De Somer F, Pattyn P. Safety and efficacy of hyperthermic intraperitoneal chemoperfusion with high-dose oxaliplatin in patients with peritoneal carcinomatosis. Ann Surg Oncol. 2008;15(2):535-41.

19. Raymond E, Chaney S, Taamma A, Cvitkovic E. Oxaliplatin: a review of preclinical and clinical studies. Ann Oncol. 1998;9(10):1053-71.

20. Elias D, Bonnay M, Puizillou JM, et al. Heated intra-operative intraperitoneal oxaliplatin after complete resection of peritoneal carcinomatosis: pharmacokinetics and tissue distribution. Ann Oncol. 2002;13(2):267-72.

21. Mehta AM, Huitema AD, Burger JW, Brandt-Kerkhof AR, van den Heuvel SF, Verwaal VJ. Standard clinical protocol for bidirectional hyperthermic intraperitoneal chemotherapy (HIPEC): systemic leucovorin, 5-fluorouracil, and heated intraperitoneal oxaliplatin in a chloride-containing carrier solution. Ann Surg Oncol. 2017;24(4):990-7.

22. Allen TM, Cullis PR. Liposomal drug delivery systems: from concept to clinical applications. Adv Drug Deliv Rev. 2013;65(1):36-48.

23. Dakwar GR, Shariati M, Willaert W, Ceelen W, De Smedt SC, Remaut K. Nanomedicine-based intraperitoneal therapy for the treatment of peritoneal carcinomatosis-mission possible? $A d v$ Drug Deliv Rev. 2017;108:13-24.

24. Jerremalm E, Wallin I, Ehrsson H. New insights into the biotransformation and pharmacokinetics of oxaliplatin. J Pharm Sci. 2009;98(11):3879-85.

25. Graham MA, Lockwood GF, Greenslade D, Brienza S, Bayssas M, Gamelin E. Clinical pharmacokinetics of oxaliplatin: a critical review. Clin Cancer Res. 2000;6(4):1205-18. 